\title{
Influence of a Supercritical Electric Dipole Moment on the Photodetachment of $\mathrm{C}_{3} \mathrm{~N}^{-}$
}

\author{
Malcolm Simpson®, Markus Nötzold, Tim Michaelsen, Robert Wild $\odot$, Franco A. Gianturco, and Roland Wester ${ }^{*}$ \\ Institut für Ionenphysik und Angewandte Physik, Universität Innsbruck, Technikerstraße 25, 6020 Innsbruck, Austria
}

(Received 5 February 2021; revised 28 April 2021; accepted 26 May 2021; published 19 July 2021)

\begin{abstract}
Threshold photodetachment spectroscopy of the molecular ion $\mathrm{C}_{3} \mathrm{~N}^{-}$has been performed at both 16(1) and 295(2) $\mathrm{K}$ in a 22-pole ion trap. The 295(2) K spectrum shows a large increase in the cross section with an onset about $200 \mathrm{~cm}^{-1}$ below threshold, which is explained by significant vibrational excitation of the trapped ions at room temperature. This excitation disappears at cryogenic temperatures leading to an almost steplike onset of the cross section at threshold, which cannot be adequately described with a Wigner threshold law. Instead, we show that the model developed by O'Malley for photodetachment from neutrals with large permanent dipoles [Phys. Rev. 137, A1668 (1965)] fits very well to the data. A high-resolution scan of the threshold region yields additional features, which we assign to the rotational $P$ and $R$ branches of an electronic transition to a dipole-bound state with ${ }^{1} \Sigma^{+}$symmetry. This state is found $2(1) \mathrm{cm}^{-1}$ below threshold in very good agreement with a recent computational prediction. We furthermore refine the value of the electron affinity of $\mathrm{C}_{3} \mathrm{~N}$ to be $34727(1) \mathrm{cm}^{-1}$.
\end{abstract}

DOI: 10.1103/PhysRevLett.127.043001

The interaction between free electrons and neutral atoms or molecules is a fundamental process that plays a role in many areas of physics, including gas discharges, planetary atmospheres, and interstellar environments [1-4]. For photodetachment from atomic negative ions the cross section near threshold is well described by the Wigner threshold law $k^{2 l+1}$, where $k$ is the linear momentum and $l$ the lowest allowed partial wave of the outgoing electron [5]. In 1965, O'Malley considered the case for a neutral molecule supporting a permanent electric dipole moment and showed that a generalized Wigner threshold law only applies for weak dipoles, whereas for strong enough dipole moments the cross section oscillates about a mean value [6]. This behavior has been considered in theoretical studies [7], but has so far not been observed in experiment.

The inverse process of photodetachment is radiative electron attachment (REA) in which a neutral molecule collides with a free electron and relaxes through radiative emission to a valence bound anionic state. This is assumed to be an important mechanism for the formation of interstellar molecular anions [8,9], which have been speculated to exist for about 50 years [10] and successfully discovered a little over a decade ago [11-21]. To quantitatively model anion abundances in interstellar molecular clouds, accurate rate constants for formation and destruction are required [4]. Our group has previously obtained

Published by the American Physical Society under the terms of the Creative Commons Attribution 4.0 International license. Further distribution of this work must maintain attribution to the author(s) and the published article's title, journal citation, and DOI. absolute photodetachment cross sections for $\mathrm{C}_{n} \mathrm{H}^{-}(n=2$, $4,6)$ [22] and $\mathrm{C}_{n} \mathrm{~N}^{-}(n=1,3)$ [23]. In the latter work it was shown that photodetachment is a primary destruction mechanism for negative ions in the circumstellar envelope IRC +10216 . These results have been used to benchmark calculations for REA and it was shown that the process is too slow by many orders of magnitude to explain the observed abundances of $\mathrm{CN}^{-}$and $\mathrm{C}_{3} \mathrm{~N}^{-}$in interstellar clouds [24].

Highly polar neutral molecules, with a permanent electric dipole moment greater than $\sim 1.67 \mathrm{D}$, referred to as supercritical, can support dipole-bound states (DBS) within a few meV of the electron detachment threshold [25]. Such DBS may be observed spectroscopically [2630] or in electron scattering [2]. It has been proposed that formation rates of interstellar anions due to REA may be augmented by the incoming electron transitioning through such weakly bound states before relaxing to the ground state [31,32]. In fact, photodetachment spectroscopy of $\mathrm{C}_{n} \mathrm{H}^{-}(n=4,6,8)$ has yielded a series of features above and below the detachment threshold [29]. For $n=6$ and 8 the features were attributed to DBSs caused by the interaction of the excess electron with the large permanent dipole moments of $\mathrm{C}_{6} \mathrm{H}$ and $\mathrm{C}_{8} \mathrm{H}$. In $\mathrm{C}_{4} \mathrm{H}^{-}$, the DBSs were attributed to the interaction with the low-lying ${ }^{2} \Pi$ excited state. Recently, a DBS with $\pi$-type symmetry has been observed in a resonant two-photon measurement of 9-anthrol ( $d=3.6 \mathrm{D})$ anions [30]. Transitions to the dipole forbidden $\pi$ state are explained by population transfer from a near degenerate $\sigma$-type DBS which is accessed by the laser [30]. Furthermore, a pump-probe scheme combined with photoelectron imaging has been used to measure the 

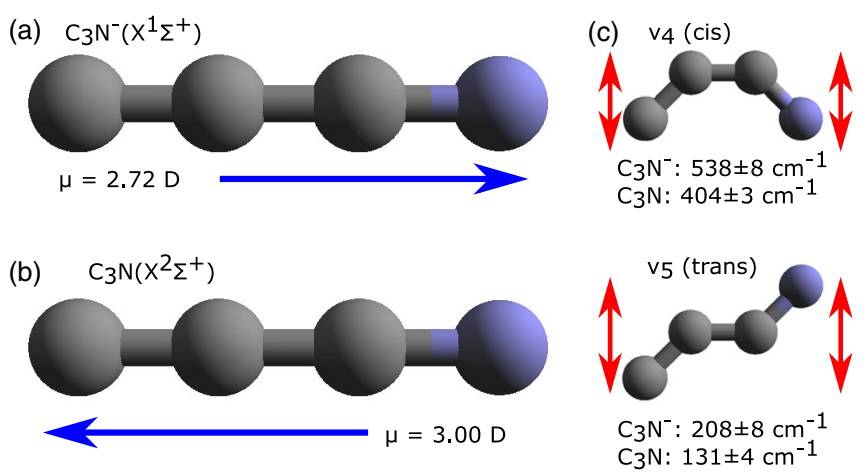

FIG. 1. (a) Structure of $\mathrm{C}_{3} \mathrm{~N}^{-}$in its ${ }^{1} \Sigma^{+}$electronic ground state with a permanent dipole moment of $d=2.72 \mathrm{D}$ [32] and a negative charge located mainly on the carbon atom. (b) Neutral $\mathrm{C}_{3} \mathrm{~N}$ in its ${ }^{2} \Sigma^{+}$ground state with a permanent dipole of $d=3.00 \mathrm{D}$ [32], which is flipped by $180^{\circ}$ with respect to the anion. (c) The doubly degenerate cis and trans vibrational bending modes in the two molecules. The harmonic frequencies are taken from Refs. [34,36].

autodetachment lifetimes of the excited vibrational Feshbach resonances of a DBS in phenoxide [33].

In this Letter we present near threshold photodetachment spectroscopy of the interstellar anion $\mathrm{C}_{3} \mathrm{~N}^{-}$in a 22-pole ion trap. The continuum cross section is well modeled by the prediction of O'Malley [6]. Just below the $\mathrm{C}_{3} \mathrm{~N}+e^{-}$ threshold we observe the resolved rotational contour of a dipole-bound state, which has the proper characteristics to be the gateway to forming interstellar $\mathrm{C}_{3} \mathrm{~N}^{-}$. Details of the target system are given in Fig. 1. Photoelectron spectroscopy of $\mathrm{C}_{3} \mathrm{~N}^{-}$has previously been performed using slow electron velocity-map imaging, which yielded the current best determination of the electron affinity for the $\mathrm{C}_{3} \mathrm{~N}$ molecule of $34718 \pm 8 \mathrm{~cm}^{-1}$ [34]. $\mathrm{C}_{3} \mathrm{~N}$ has a supercritical permanent dipole of $3.00 \mathrm{D}$ [32]. A dipole-bound state has been predicted to lie about $2 \mathrm{~cm}^{-1}$ below the detachment threshold [35].

Our 22-pole ion trap experiment has been presented previously $[22,37,38]$. Here we give the important details for the present measurements. $\mathrm{C}_{3} \mathrm{~N}^{-}$is produced in a pulsed plasma discharge in a supersonic expansion of acetonitrile in an argon carrier gas. A set of Wiley-McLaren style electrodes accelerate all negatively charged species along the first axis of the experiment to $500 \mathrm{eV}$ of kinetic energy. A $90^{\circ}$ quadrupole bender passes the ions toward the ion trap, which consists of 22 stainless steel rods for the radial confinement and hollow cylindrical end-cap electrodes for the axial confinement. The trap is in thermal contact with its copper housing and fixed to a closed-cycle helium cryostat that allows temperatures to be adjusted between room temperature and about $10 \mathrm{~K}$. The trap electrodes, including the radio frequency rods, are referenced to a dc offset voltage of about $-500 \mathrm{~V}$, with a small additive voltage being applied to the end-cap electrodes. $\mathrm{C}_{3} \mathrm{~N}^{-}$ions are decelerated and loaded into the trap through the end-cap electrodes at the trap entrance. Lighter and heavier masses are separated by time of flight and are blocked by pulsing the end-cap voltage. A few thousand ions are trapped and then thermalized to the trap temperature through application of helium buffer gas, which is estimated to occur within a few tens of milliseconds. In the present measurements we have therefore implemented a pretrapping period of $300 \mathrm{~ms}$ prior to interaction with the photodetachment laser.

Ultraviolet radiation for photodetachment is produced by a frequency-doubled pulsed dye laser (Radiant Dyes Narrow Scan) pumped by the second harmonic of a Nd: YAG laser with $50 \mathrm{~Hz}$ repetition rate (Spectra Physics Quanta-Ray). The bandwidth of the dye fundamental was measured to be $0.35(5) \mathrm{cm}^{-1}$ and we estimate the bandwidth of the frequency-doubled UV pulse to be twice this value. The UV laser beam enters the vacuum chamber through a fused silica window and traverses the trap through the end-cap electrodes. Its relative pulse energy is measured by a bolometer positioned outside the opposite end of the vacuum chamber. The laser beam is opened by a mechanical shutter after pretrapping and is closed after $10 \mathrm{~s}$ of interaction with the trapped ions and $0.1 \mathrm{~s}$ prior to their extraction. The ions are extracted from the trap by once again pulsing the entrance end cap and guided toward a microchannel plate (MCP) detector. After a trapping cycle with laser interaction a cycle with $0.1 \mathrm{~s}$ trapping time is performed as background measurement. After five repetitions the laser frequency is stepped forward. Given that ion depletion is weak, the photodetachment signal is determined by subtracting the laser-depleted MCP ion signal from the background signal, followed by normalization to the laser pulse energy.

Threshold photodetachment spectra of $\mathrm{C}_{3} \mathrm{~N}^{-}\left(X^{1} \Sigma^{+}\right)+$ $h \nu \rightarrow \mathrm{C}_{3} \mathrm{~N}\left(X^{2} \Sigma^{+}\right)+e^{-}$have been measured at both 16(1) and 295(2) $\mathrm{K}$ in the 22-pole ion trap. Figure 2 shows the relative photodetachment cross sections for both temperatures as a function of photon energy with a step size of $2 \mathrm{~cm}^{-1}$. The black vertical dashed lines mark the electron affinity obtained in this work (see below), which agrees within the error bar with the previously determined value [34]. At this laser frequency a clear increase in the cross section is observed.

In the room temperature measurement (Fig. 2, upper panel), we observe considerable signal commencing about $200 \mathrm{~cm}^{-1}$ below threshold. This can only be attributed to internal excitation of the trapped anions. Rotational excitation alone could only explain detachment that starts about $30 \mathrm{~cm}^{-1}$ below threshold. Instead, vibrational excitation of the two lowest energy bending modes of $\mathrm{C}_{3} \mathrm{~N}^{-}, \nu_{4}$ and $\nu_{5}$ (see Fig. 1), is responsible. They require 538(8) and $208(8) \mathrm{cm}^{-1}$ for activation, respectively [34], and carry a population of around $28 \%(16 \%, 8 \%)$ in the first (second, third) excited $\nu_{5}$ level and $6 \%$ in the first $\nu_{4}$ level at 


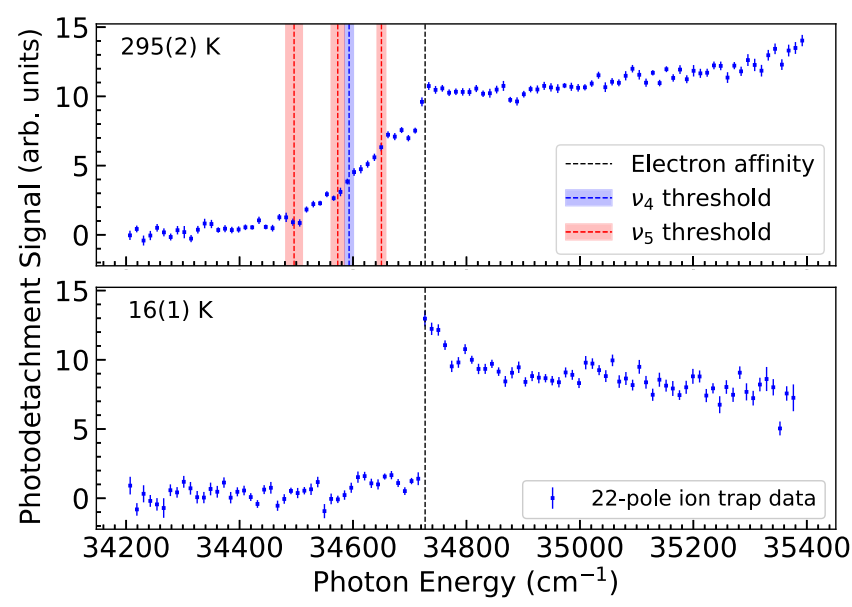

FIG. 2. Relative photodetachment cross section of trapped $\mathrm{C}_{3} \mathrm{~N}^{-}$at $295(2) \mathrm{K}$ (upper panel) and 16(1) $\mathrm{K}$ (lower panel) buffer gas. Error bars denote $1 \sigma$ uncertainties. The electron affinity determined in this work, the detachment threshold for rovibrational ground state anions, is shown as the vertical dashed black lines. In the upper panel the thresholds for anions in their two lowest excited bending modes $\nu_{4}$ and $\nu_{5}$, assuming $\Delta v=0$ transitions, are shown as blue and red vertical dashed lines (shaded areas show the associated errors).

room temperature. Photodetachment transitions from these excited $\nu_{4}$ and $\nu_{5}$ levels are expected to follow a $\Delta v=0$ propensity rule given that the molecular structure remains essentially unchanged. The respective detachment thresholds are shown in Fig. 2 as the blue and red vertical dashed lines. They appear in good agreement with smaller steps in the detachment signal and explain the detachment signal below threshold very well.

The lower panel of Fig. 2 shows the identical scan range taken at a trap temperature of 16(1) K. The vibrational excitation has completely disappeared as expected for anions in the vibrational ground state only. This results in a sharp threshold in excellent agreement with the previous $\mathrm{C}_{3} \mathrm{~N}$ electron affinity assignment by Yen et al. [34]. Of particular note is the curvature of the cross section above threshold, which cannot be modeled by a modified Wigner law [5] of the form

$$
\sigma_{\mathrm{Wigner}}(\epsilon) \propto \epsilon^{P} .
$$

Here $\epsilon$ is the kinetic energy of the outgoing electron and $P$ is a positive real number that is dependent on the partial wave of the outgoing electron and any permanent dipole moment of the neutral molecule. In Ref. [39] we presented a model detailing the value of the $P$ exponent for different molecular symmetries and dipole moments. However, for the size of the $\mathrm{C}_{3} \mathrm{~N}$ dipole moment of $3.00 \mathrm{D}$, this model and the Wigner threshold law are no longer applicable, which is confirmed by the data.

In 1965 O'Malley [6] presented a more specific model for the photodetachment cross section for highly polar molecules of the form

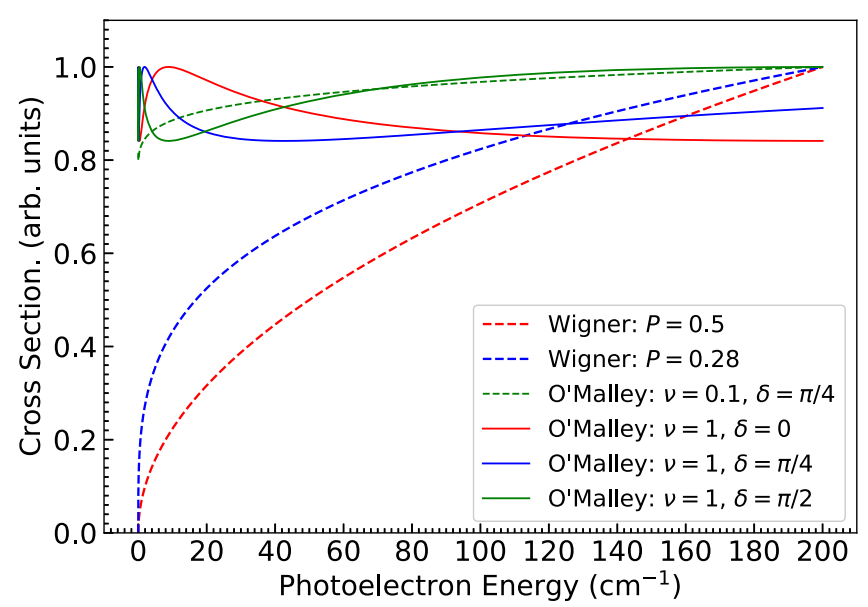

FIG. 3. Simulated energy dependence of the Wigner [Eq. (1)] and O'Malley [Eq. (2)] cross sections for a range of parameter values (normalized to a maximum of 1). The red dashed curve shows Wigner's law for $s$-wave detachment from a nonpolar core $(P=0.5)$ and the blue dashed curve shows the modified cross section for the hydroxyl anion $\mathrm{OH}^{-}$. The $\mathrm{OH}$ neutral supports a barely subcritical permanent dipole of $1.66 \mathrm{D}$ [40]. The O'Malley cross section [Eq. (2)] bears similarity with the Wigner cross section for a small value of $\nu=0.1$ (green dashed line), while for $\nu=1$ the cross section shows oscillatory behavior that depends on the phase shift $\delta$ (solid lines).

$\sigma_{\mathrm{O}^{\prime} \text { Malley }}(\epsilon) \propto\left[\sinh ^{2}\left(\frac{\nu \pi}{2}\right)+\cos ^{2}\left(\frac{\nu}{2} \ln 2 \epsilon+\delta\right)\right]^{-1}$,

where $\nu=\left[d-(l+1 / 2)^{2}\right]^{1 / 2}$ is real valued and a function of the permanent dipole $d$ (in atomic units) and the partial wave of the outgoing electron $l . \epsilon$ is again the electron energy (in atomic units) and $\delta$ a phase factor that is sensitive to the short-range behavior of the potential. With the $\mathrm{C}_{3} \mathrm{~N}$ dipole and since $\mathrm{C}_{3} \mathrm{~N}^{-}$predominantly undergoes $s$-wave detachment $(l=0), \nu$ takes the value 0.96 . A comparison between the energy dependence of the Wigner and O'Malley cross sections for different values of $\nu$ and $\delta$ is shown in Fig. 3. The comparison with the cold ion data reveals a good qualitative agreement with the O'Malley cross section for $\nu=1$.

In Fig. 4 we show a high-resolution scan over the threshold region with a step size of $0.2 \mathrm{~cm}^{-1}$. Two notable peaks become apparent at threshold, which we assign to the $P$ and $R$ branches of a two-photon detachment process via a dipole-bound state. Similar DBS features have been found in photodetachment spectroscopy of $p$ - $\mathrm{FC}_{6} \mathrm{H}_{4} \mathrm{O}^{-}$[41]. In Ref. [35], it was reported that at least two DBSs are expected to exist within a few $\mathrm{meV}$ of the detachment threshold of $\mathrm{C}_{3} \mathrm{~N}^{-}$. One of them is of ${ }^{1} \Sigma^{+}$symmetry and has a binding energy of about $2 \mathrm{~cm}^{-1}$. Transitions to this state from the $\mathrm{C}_{3} \mathrm{~N}^{-}$ground state are electric dipole allowed and are therefore observable in our experiment. The other DBS 


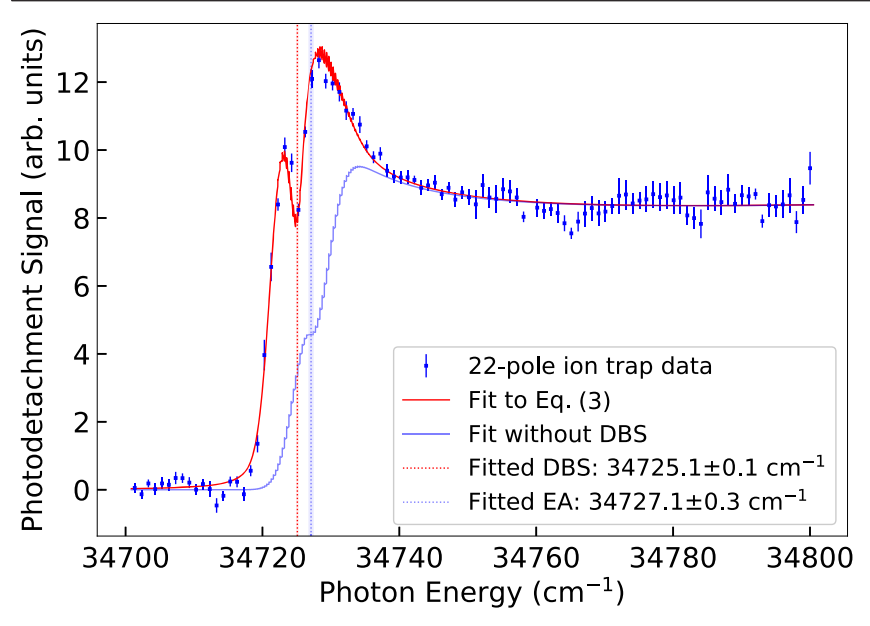

FIG. 4. High-resolution relative photodetachment cross section of $\mathrm{C}_{3} \mathrm{~N}^{-}$at $16(1) \mathrm{K}$ trap temperature. Two features in the threshold region are visible, attributed to the $P$ and $R$ rotational branches of the excitation to a dipole-bound state with ${ }^{1} \Sigma^{+}$ symmetry. The model given in Eq. (3) fits very well to the data (red line), which yields the denoted rotational origin of the dipole-bound state and the $\mathrm{C}_{3} \mathrm{~N}$ electron affinity. The blue line is the contribution of the nonresonant cross section alone.

is of ${ }^{3} \Sigma^{+}$symmetry and not accessible via electric dipole transitions.

In order to quantitatively confirm our assignment, we model the photodetachment cross section as a sum of the two-photon excitation to the DBS and the single-photon detachment of Eq. (2). We initially compute the electronic transition to the DBS using the PGOPHER software package [42], which yields the temperature-dependent, normalized rotational line list. The rotational constants for $\mathrm{C}_{3} \mathrm{~N}$ and $\mathrm{C}_{3} \mathrm{~N}^{-}, B^{\prime}=0.165 \mathrm{~cm}^{-1}$ and $B^{\prime \prime}=0.162 \mathrm{~cm}^{-1}$, were taken from Refs. $[43,44]$. Note that due to the folding of the lines with the laser bandwidth of $\sim 0.7 \mathrm{~cm}^{-1}$, full rotational resolution is not possible here and only the envelopes of the $P$ and $R$ branches are observable. Given that the rotational temperature of the trapped ion ensemble often does not fully thermalize with the trap temperature [45], the analysis has been performed over a range of temperatures with the best fit occurring at $25 \mathrm{~K}$, well within the expected range $[45,46]$. To model the single-photon component we modify the fit function that we have used to describe the threshold photodetachment of $\mathrm{CN}^{-}$[38] by replacing the Wigner law with that of O'Malley [Eq. (2)]. Combining the foregoing yields a fit function of the form

$$
\begin{aligned}
\sigma= & \sum_{J^{\prime}, J^{\prime \prime}}\left[a S_{J^{\prime \prime}}^{J^{\prime}}(T) \frac{\gamma^{2}}{\left(E_{\mathrm{ph}}-E_{J^{\prime \prime} J^{\prime}}^{\mathrm{DBS}}\right)^{2}+\gamma^{2}}\right. \\
& \left.+b W_{J^{\prime \prime}}(T)\left|C_{J^{\prime \prime} 010}^{J^{\prime} 0}\right|^{2} \sigma_{\mathrm{O}^{\prime} \text { Malley }}\left(E_{\mathrm{ph}}-E_{J^{\prime \prime} J^{\prime}}^{E A}\right)\right],
\end{aligned}
$$

where $S_{J^{\prime \prime}}^{J^{\prime}}(T)$ is used to denote the temperature-dependent line strength for the transition $J^{\prime \prime} \rightarrow J^{\prime}$ to the DBS at energy $E_{J^{\prime \prime} J^{\prime}}^{\mathrm{DBS}}, \gamma$ is the individual Lorentzian linewidth, $W_{J^{\prime \prime}}(T)$ is the thermal rotational population in the anion, and $\left|C_{J^{\prime \prime} 010}^{J^{\prime}}\right|^{2}$ are Hönl-London factors. $E_{J^{\prime \prime} J^{\prime}}^{E A}$ is the rotational statedependent detachment threshold and $E_{\mathrm{ph}}$ the photon energy. $a$ and $b$ are proportionality factors for the twophoton and single-photon detachment components, respectively.

Equation (3) has been fitted to the high-resolution data using a nonlinear multiparameter fit. The result is shown in Fig. 4 as the red curve, which shows an excellent agreement with the measured relative cross section. This yields the position of the rotational origin of the dipole-bound state at $34725.1 \pm 0.1_{\text {stat }} \pm 0.2_{\text {syst }} \mathrm{cm}^{-1}$ above the ground state and an improved determination of the $\mathrm{C}_{3} \mathrm{~N}$ electron affinity of $34727.1 \pm 0.3_{\text {stat }} \pm 0.6_{\text {syst }} \mathrm{cm}^{-1}$ and a phase shift $\delta=0.5$. The quoted statistical errors have been determined from a reduced $\chi^{2}$ analysis. They are highlighted in Fig. 4 as the red and blue dashed lines and shaded regions, respectively. We have estimated the additionally given systematic errors to account for possible imperfections of the fit model. Taken together the binding energy of the DBS with respect to neutral $\mathrm{C}_{3} \mathrm{~N}$ is $2 \pm 1 \mathrm{~cm}^{-1}$, which is in very good agreement with the computational prediction [35]. The fit yields a linewidth of $\gamma=1.4 \mathrm{~cm}^{-1}$, which is larger than the laser linewidth and suggests that the excitation is significantly saturated. This can be well explained by our computational estimate of the transition matrix element of about $1 \mathrm{D}$. The extracted energy dependence of the O'Malley cross section determined from the final fit parameters is shown as the blue curve. We did not observe an intensity dependence of the two-photon contribution to the cross section, which is another indication that the bound-bound transition is strongly saturated.

We have shown that the supercritical electric dipole moment of $\mathrm{C}_{3} \mathrm{~N}$ strongly modifies the photodetachment cross section near threshold as predicted theoretically more than 50 years ago [6]. In addition, we have observed a dipole-bound state in $\mathrm{C}_{3} \mathrm{~N}^{-}$, which may present a pathway for the enhancement of radiative electron attachment through an initial trapping of the incoming electron in the DBS followed by a further decay to the compact valence bound state as predicted earlier [32]. In the DBS, the weakly bound wave function of this extra electron is deemed to be one or more orders of magnitude larger than that of the deeply bound anionic state $[32,35]$. Hence, one expects that the corresponding square of the transition matrix element causes the final REA rate coefficient to become substantially larger. Specific calculations will be required to verify if this can explain the observed abundance of $\mathrm{C}_{3} \mathrm{~N}^{-}$in interstellar molecular clouds. 
This work has been supported by the Austrian Science Fund (FWF) through the Doctoral Programme Atoms, Light, and Molecules, Project No. W1259-N27, and through Project No. P27047-N20.

*roland.wester@uibk.ac.at

[1] H. Hotop, M.-W. Ruf, M. Allan, and I. I. Fabrikant, Adv. At. Mol. Opt. Phys. 49, 85 (2003).

[2] I. I. Fabrikant, J. Phys. B 49, 222005 (2016).

[3] L. Campbell and M. J. Brunger, Int. Rev. Phys. Chem. 35, 297 (2016).

[4] T. J. Millar, C. Walsh, and T. A. Field, Chem. Rev. 117, 1765 (2017).

[5] E. P. Wigner, Phys. Rev. 73, 1002 (1948).

[6] T. F. O’Malley, Phys. Rev. 137, A1668 (1965).

[7] N. Douguet, V. Kokoouline, and A. E. Orel, Phys. Rev. A 90, 063410 (2014).

[8] P. J. Sarre, J. Chim. Phys. Phys.-Chim. Biol. 77, 769 (1980).

[9] E. Herbst, Nature (London) 289, 656 (1981).

[10] A. Dalgarno and R. A. McCray, Astrophys. J. 181, 95 (1973).

[11] M. C. McCarthy, C. A. Gottlieb, H. Gupta, and P. Thaddeus, Astrophys. J. 652, L141 (2006).

[12] J. Cernicharo, M. Guélin, M. Agúndez, K. Kawaguchi, M. McCarthy, and P. Thaddeus, Astron. Astrophys. 467, L37 (2007).

[13] N. Sakai, T. Sakai, and S. Yamamoto, Astrophys. J. 673, L71 (2008).

[14] M. Agúndez, J. Cernicharo, M. Guélin, M. Gerin, M. C. McCarthy, and P. Thaddeus, Astron. Astrophys. 478, L19 (2008).

[15] M. A. Cordiner, J. V. Buckle, E. S. Wirström, A. O. H. Olofsson, and S. B. Charnley, Astrophys. J. 770, 48 (2013).

[16] S. Brünken, H. Gupta, C. A. Gottlieb, M. C. McCarthy, and P. Thaddeus, Astrophys. J. Lett. 664, L43 (2007).

[17] A. J. Remijan, J. M. Hollis, F. J. Lovas, M. A. Cordiner, T. J. Millar, A. J. Markwick-Kemper, and P. R. Jewell, Astrophys. J. Lett. 664, L47 (2007).

[18] M. Agúndez, J. Cernicharo, M. Guélin, C. Kahane, E. Roueff, J. Kłos, F. J. Aoiz, F. Lique, N. Marcelino, J. R. Goicoechea, M. G. Garcia, C. A. Gottlieb, M. C. McCarthy, and P. Thaddeus, Astron. Astrophys. 517, L2 (2010).

[19] P. Thaddeus, C. A. Gottlieb, H. Gupta, S. Brunken, M. C. McCarthy, M. Agndez, M. Guaulin, and J. Cernicharo, Astrophys. J. 677, 1132 (2008).

[20] J. Cernicharo, N. Marcelino, J. R. Pardo, M. Agúndez, B. Tercero, P. de Vicente, C. Cabezas, and C. Bermúdez, Astron. Astrophys. 641, L9 (2020).

[21] J. Cernicharo, M. Guélin, M. Agúndez, M. McCarthy, and P. Thaddeus, Astrophys. J. Lett. 688, L83 (2008).

[22] T. Best, R. Otto, S. Trippel, P. Hlavenka, A. von Zastrow, S. Eisenbach, S. Jezouin, R. Wester, E. Vigren, M. Hamberg, and W. D. Geppert, Astrophys. J. 742, 63 (2011).
[23] S. S. Kumar, D. Hauser, R. Jindra, T. Best, Š. Roučka, W. D. Geppert, T. J. Millar, and R. Wester, Astrophys. J. 776, 25 (2013).

[24] M. Khamesian, N. Douguet, S. Fonseca dos Santos, O. Dulieu, M. Raoult, W. J. Brigg, and V. Kokoouline, Phys. Rev. Lett. 117, 123001 (2016).

[25] R. F. Wallis, R. Herman, and H. W. Milnes, J. Mol. Spectrosc. 4, 51 (1960).

[26] R. L. Jackson, P. C. Hiberty, and J. I. Brauman, J. Chem. Phys. 74, 3705 (1981).

[27] K. R. Lykke, R. D. Mead, and W. C. Lineberger, Phys. Rev. Lett. 52, 2221 (1984).

[28] C. Desfranćois, H. Abdoul-Carime, N. Khelifa, and J. P. Schermann, Phys. Rev. Lett. 73, 2436 (1994).

[29] T. Pino, M. Tulej, F. Güthe, M. Pachkov, and J. P. Maier, J. Chem. Phys. 116, 6126 (2002).

[30] D.-F. Yuan, Y. Liu, C.-H. Qian, Y.-R. Zhang, B. M. Rubenstein, and L.-S. Wang, Phys. Rev. Lett. 125, 073003 (2020).

[31] F. Carelli, M. Satta, T. Grassi, and F. A. Gianturco, Astrophys. J. 774, 97 (2013).

[32] F. Carelli, F. Gianturco, R. Wester, and M. Satta, J. Chem. Phys. 141, 054302 (2014).

[33] D. H. Kang, S. An, and S. K. Kim, Phys. Rev. Lett. 125, 093001 (2020).

[34] T. A. Yen, E. Garand, A. T. Shreve, and D. M. Neumark, J. Phys. Chem. A 114, 3215 (2010).

[35] S. V. Jerosimic, M. Z. Milovanovic, R. Wester, and F. A. Gianturco, Adv. Quantum Chem. 80, 47 (2019).

[36] K. Hoshina and Y. Endo, J. Chem. Phys. 127, 184304 (2007).

[37] R. Wester, J. Phys. B 42, 154001 (2009).

[38] M. Simpson, M. Nötzold, A. Schmidt-May, T. Michaelsen, B. Bastian, J. Meyer, R. Wild, F. A. Gianturco, M. Milovanović, V. Kokoouline, and R. Wester, J. Chem. Phys. 153, 184309 (2020).

[39] O. Lakhmanskaya, M. Simpson, S. Murauer, M. Nötzold, E. Endres, V. Kokoouline, and R. Wester, J. Chem. Phys. 149, 104302 (2018).

[40] K. Peterson, G. Fraser, and W. Klemperer, Can. J. Phys. 62, 1502 (1984).

[41] C.-H. Qian, G.-Z. Zhu, and L.-S. Wang, J. Phys. Chem. Lett. 10, 6472 (2019).

[42] C. M. Western, J. Quant. Spectrosc. Radiat. Transfer 186, 221 (2017).

[43] M. C. McCarthy, G. W. Fuchs, J. Kucera, G. Winnewisser, and P. Thaddeus, J. Chem. Phys. 118, 3549 (2003).

[44] M. C. McCarthy and P. Thaddeus, J. Chem. Phys. 129, 054314 (2008).

[45] E. S. Endres, G. Egger, S. Lee, O. Lakhmanskaya, M. Simpson, and R. Wester, J. Mol. Spectrosc. 332, 134 (2017).

[46] O. Lakhmanskaya, M. Simpson, and R. Wester, Phys. Rev. A 102, 012809 (2020). 